\title{
A Novel Method for Quantifying the Composition of Mineralogical Phase in Iron Ore Sinter
}

\author{
Xuewei LV, Chenguang BAI, Guibao QIU, Shengfu ZHANG and Ruimeng SHI \\ College of Materials Science \& Engineering, Chongqing University, Chongqing, 400044, P.R. China. \\ E-mail: Ivxuewei@163.com (Lv), bguang@cqu.edu.cn (Bai)
}

(Received on November 26, 2008; accepted on February 9, 2009)

\begin{abstract}
This paper presents a new method to calculate accurately the composition of the mineralogical phase in iron ore sinter, especially the minor phases. This model is based on the combined model of gray-level distribution for a multi-phase system. The composition of the mineralogical phase is calculated by minimizing the difference between the theoretical gray-level histogram and the actual gray-level histogram of a micrograph of the iron ore sinter. A genetic algorithm and an improved genetic algorithm are introduced to solve this extreme value problem. Suitable parameters for the algorithm were determined by comparing the solutions for each of several cases. The example provided demonstrates that this model is valid, especially for the minor phases, and the solution is credible.
\end{abstract}

KEY WORDS: composition of mineralogical phase; genetic algorithm; gray-level histogram; quantification; iron ore sinter.

\section{Introduction}

Accurately quantifying the composition of mineralogical phase is important for improving the quality of iron ore sinter. Especially in recent years, the iron ore resources have decreased dramatically worldwide. Many iron and steel companies face a shortage of high grade iron ore for sintering. As a result, many low grade, compositionally complex ores are used in the sinter plant. It is crucial, therefore, to control the properties of iron ore sinter and maintain the stability of the blast furnace. This objective requires accurate quantification of the composition of the mineralogical phases. Two systems have been developed previously to measure mineralogy phases. The first, developed by IRSID and the School of Mines of Paris ${ }^{1,2}$ is based on operational morphology. The second, developed by University of Tokyo and Nippon Corporation, ${ }^{3)}$ is based on pixel statistics.

Jeulin determined the composition of the mineralogical phase by calculating the phase area after one pixel erosion. ${ }^{2)}$ Shibuya determined the area of phase by counting the pixels of the phase in an image. ${ }^{3)}$ Both these systems rely on the same theory to determine the fraction of the image represented by each mineralogical phase. That is, they assume that the gray level represents the reflective power of the mineralogical phases. However, no research confirms the relationship between the gray level of the image and the reflective power of the mineralogical phases. Furthermore, neither system explains how the range of the gray level is determined for mineralogical phases.

It is by no means certain, however, that the range of gray level is continuous, with no overlap. Generally, the range of reflective power of a mineralogy phase is constant because of the various crystallizing conditions, as shown in Table $\mathbf{1 .}$ According to the principle expressed in Eq. (1), ${ }^{4)}$ if the coefficient $k$ is defined, the gray level $I$ should be a fixed value when the reflective power $R$ is fixed:

$$
R=k \times \frac{I}{255}
$$

where $k$ is a coefficient representing the relationship between $R$ and $I$ and determined by the means of image capture, signal processing, or $\mathrm{A} / \mathrm{D}$ transfer. In fact, the gray level of a mineralogy phase in a digital image is always distributed over a range. For example, a pore, whose reflective power should be 0 , distributes its gray level in a range of 20-50 (with 256 being the highest gray level in a 24-bit RGB file). This distribution is largely because the signal is discrete when it is transferred from analog to digital. The various parts of the scene interact with each other as the image forms. As a result, these interactions are captured in the image created. The assumption that gray-level ranges for the mineralogical phase are continuous and have no overlap is, therefore, invalid. Another paper ${ }^{4)}$ by the same authors discusses the relationship between the mineralographic features of sinter ore and its gray histogram. It also develops the Gaussian normal distribution model of the gray level for the mineralogy phase shown in the micrograph. This model forms the basis for the method intro-

Table 1. Index of reflection of common minerals in sinter ore.

\begin{tabular}{cccccc}
\hline mineral & magnetite & hematite & fayalite & $\begin{array}{c}\text { calcium } \\
\text { silicate }\end{array}$ & $\begin{array}{c}\text { calcium } \\
\text { ferrite }\end{array}$ \\
\hline index of reflection & 20 & $22 \sim 25$ & $10 \sim 12$ & $5 \sim 8$ & $18 \sim 18.5$ \\
\hline
\end{tabular}


duced here to quantify the composition of mineralogy phases in iron ore sinter.

\section{Model of Gray-level Distribution for Mineralogy Phases}

\subsection{Gray-level Histogram}

A gray-level histogram is an approximate expression of a density function of gray. It shows the frequency of gray level appearing in an image. The histogram is described as:

$$
G_{i}=\sum_{m=1}^{M} \sum_{n=1}^{N} P(i, m, n)
$$

where $M$ and $N$ refer to the total number of rows and columns respectively, and $G_{i}$ is the number of occurrences of gray level $i$. The definition of $P(i, m, n)$ is:

$$
P(i, m, n)= \begin{cases}1 & P(m, n)=i \\ 0 & P(m, n) \neq i\end{cases}
$$

where $P(m, n)$ is the gray level of the pixel whose coordination is $(m, n)$.

To accommodate images of various sizes, normalization is necessary and is expressed as:

$$
G_{i}^{\prime}=\frac{G_{i}}{T}
$$

where $G_{i}{ }^{\prime}$ is the normalization value, and $T$ is the total number of pixels.

\subsection{Gaussian Model of Gray-level Distribution}

The Gaussian model of gray-level distribution is expressed as:

$$
I \sim N\left(\mu, \sigma^{2}\right) .
$$

where $I$ represents gray distribution of a mineral phase. The values $\mu$ and $\sigma^{2}$, representing mean value and gray-level variance, can be calculated by Eqs. (6), (7), and (8).

$$
\begin{gathered}
\mu=E(I)=\sum_{i=I_{\max }}^{I_{\max }} G_{i} \times \varphi(i) \\
E\left(I^{2}\right)=\sum_{i=I_{\max }}^{I_{\max }} G_{i}^{2} \times \varphi(i) . . \\
\sigma^{2}=D(I)=(E(I))^{2}-E\left(I^{2}\right) .
\end{gathered}
$$

where $I_{\max }$ and $I_{\min }$ refer to the maximum and minimum gray level of the mineral phase, and $\varphi(i)$ is the frequency of gray $i$ appearing in a certain zone. The variable $\theta^{2}$, which is assumed to be constant for a mineral phase, can be calculated by averaging the standard samples. The regularities of gray-level distribution for mineral phases are thus calculated by Eqs. (6), (7), and (8). The results are listed in Table 2 .

\subsection{Combined model of Gray Level Distribution for Multi-phases System}

Generally, more than one phase is visible with a micro-
Table 2. Parameters of gray level distribution for common mineralogy phases.

\begin{tabular}{ccccccc}
\hline $\begin{array}{c}\text { mineralogy } \\
\text { phase }\end{array}$ & magnetite & hematite & fayalite & $\begin{array}{c}\text { calcium } \\
\text { silicate }\end{array}$ & $\begin{array}{c}\text { calcium } \\
\text { ferrite }\end{array}$ & pore \\
\hline$\mu$ & 121.1 & 137.8 & 70.6 & 52.6 & 109.5 & 43.1 \\
$\sigma^{2}$ & 34.0 & 19.6 & 67.9 & 47.5 & 128.7 & 10.9 \\
\hline
\end{tabular}

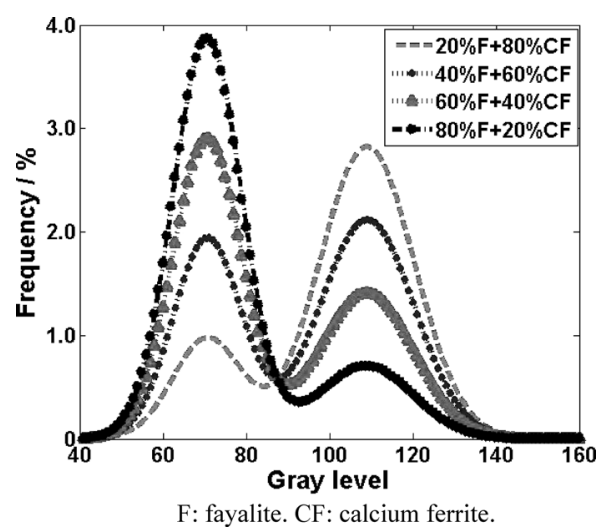

Fig. 1. Combined gray-level distribution for two phases.

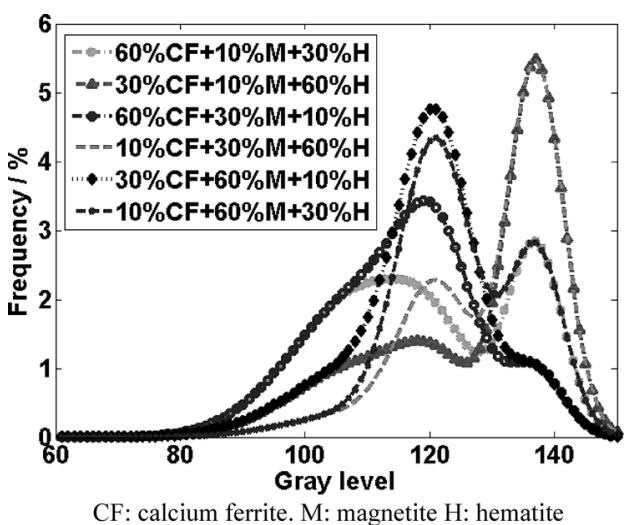

Fig. 2. Combined gray-level distribution for three phases.

scope. Therefore, the gray-level distribution for multiple phases is the sum of the density distribution functions for each phase. The combined model can be expressed as:

$$
f(x)=\sum_{i=1}^{N} \frac{C_{i}}{\sigma_{i} \sqrt{2 \pi}} e^{\frac{-\left(x-\mu_{i}\right)^{2}}{2 \sigma_{i}^{2}}}
$$

where $C_{i}$ is the fraction of the phase $i$ in the field of view. Using the distributional parameters in Table 2, Eq. (9) can be used to calculate the theoretical gray-level histogram for multiple phases.

For example, when $N=2$, the theoretical gray-level histogram for calcium ferrite and fayalite is shown in Fig. 1. This histogram changes as the fraction of each phase changes. The peak of the phase rises when the fraction of the phase increases and falls when the fraction of the phase decreases.

The theoretical gray-level histogram for calcium ferrite, magnetite, and hematite when $N=3$ is shown in Fig. 2 . 


\section{Model for Quantifying the Composition of the Min- eralogy Phase}

The model discussed above of gray-level distribution for the mineralogy phase demonstrates that the fraction of each phase influences the gray-level histogram of the micrograph. That is, the gray-level histogram reflects the composition of the mineralogical phases. Based on the combined model of gray-level distribution for a multi-phase system, the fraction of each phase can be calculated by comparing the theoretical gray-level histogram with the actual gray level histogram of a given micrograph. The difference between these two histograms changes as the composition of the mineralogical phase used for the calculation changes. The composition of the mineralogical phase can be accurately determined by continuously minimizing this difference. The equation to calculate the difference between a theoretical gray-level histogram and an actual gray level histogram can be expressed as:

$$
d=\sum_{i=1}^{i=256}\left|f(i)-G_{i}^{\prime}\right|
$$

where

$$
f(i)=\sum_{j=1}^{N} \frac{C_{j}}{\sigma_{j} \sqrt{2 \pi}} e^{\frac{-\left(x-\mu_{j}\right)^{2}}{2 \sigma_{j}^{2}}}
$$

Therefore, the calculation of the composition of the mineralogical phase is transformed so that $d$ is minimized by changing the series of $C_{j}$. The mathematical model for minimization, which refers to the object function and constraint conditions, can be expressed as:

$$
\begin{aligned}
& \text { Min } d=\sum_{i=1}^{i=256}\left|f(i)-G_{i}^{\prime}\right| \\
& \text { S.t. } 0 \leq C_{j} \leq 100 \%
\end{aligned}
$$

\section{Solving the Models through Genetic Algorithms}

Physics, Biology, Economy, and Sociology must often grapple with the classic problem of optimization. Genetic algorithms, which became popular through the work of Holland $^{5)}$ in early 1970 s, are a technique used in computing to find exact or approximate solutions to optimization and search problems.

Holland describes a genetic algorithm as follows: "Most organisms evolve by means of two primary processes: natural selection and sexual reproduction. The first determines which members of population survive and reproduce, and the second ensures mixing and recombination among the genes of their offspring. When sperm and ova fuse, matching chromosomes line up with one another and then crossover partway along their length, thus swapping genetic material. This mixing allows creatures to evolve much more rapidly than they would if each offspring simply contained a copy of the genes of a single parent, modified occasionally by mutation." Genetic algorithms are based on the knowledge of how organisms evolve, and they are categorized as global search heuristics, which are a particular

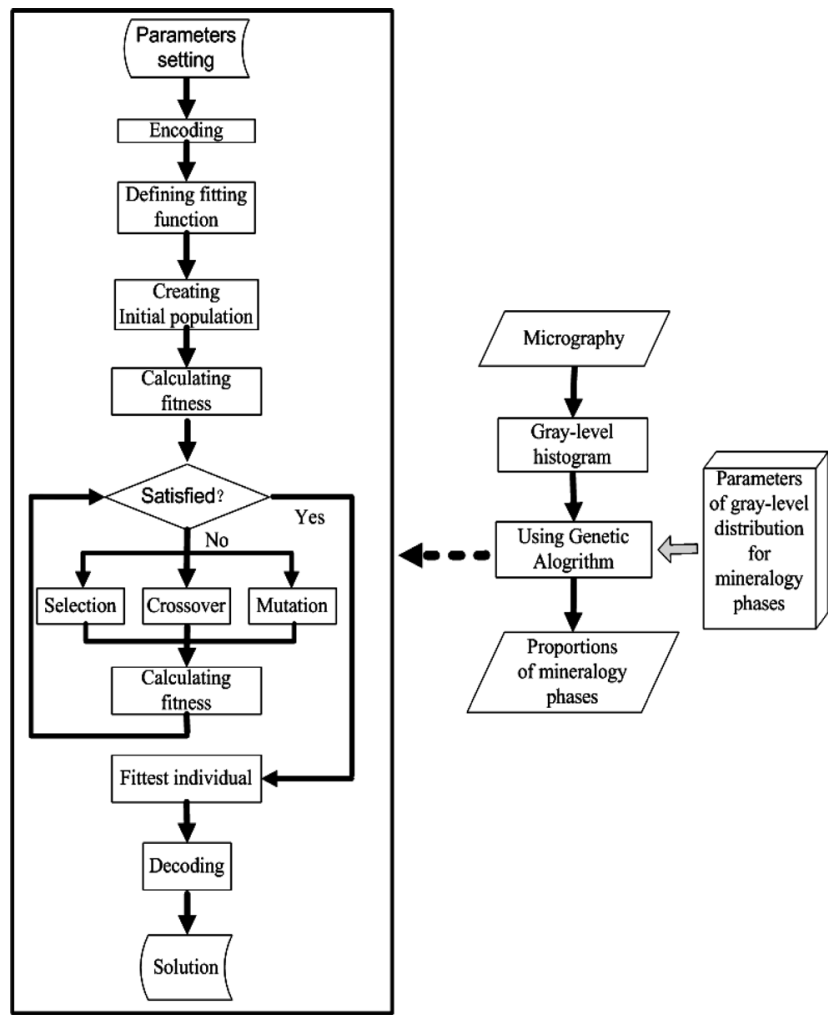

Fig. 3. Flow chart of calculating composition of mineralogy phases based on genetic algorithms.

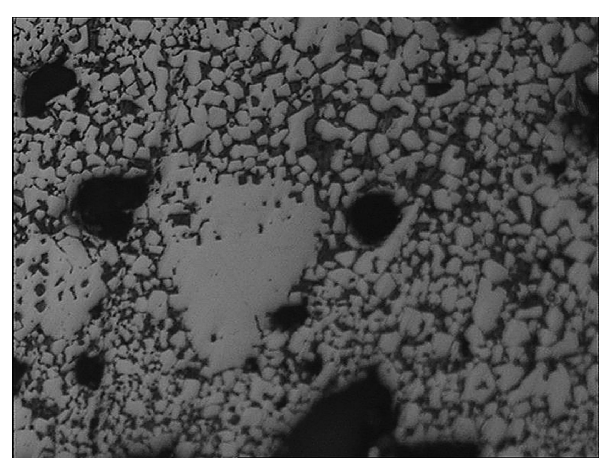

Fig. 4. Micrograph of iron ore sinter.

class of evolutionary algorithms (also known as evolutionary computation) that use techniques inspired by evolutionary biology such as inheritance, mutation, selection, and crossover (also called recombination). Nowadays, genetic algorithms are widely used to solve optimization problems.

Genetic algorithms were used to develop a program to solve Eq. (12). The flow chart of the programs is shown in Fig. 3.

\section{Application}

Taking Fig. 4 as an example, the mathematical model developed above is used, along with a genetic algorithm, to calculate the composition of each phase in the micrograph. Figure 5 shows the actual gray-level histogram of Fig. 4.

Accurate and quick results for a genetic algorithm depend on the selection of appropriate parameters. The present paper studies several cases for comparison. The principle parameters used in these cases are listed in Table $\mathbf{3}$, and 
Table 3. Parameters used in genetic algorithms.

\begin{tabular}{cccccccc}
\hline No. & $\begin{array}{c}\text { encoding } \\
\text { method }\end{array}$ & $\begin{array}{c}\text { encoding } \\
\text { length }\end{array}$ & $\begin{array}{c}\text { population } \\
\text { size }\end{array}$ & $\begin{array}{c}\text { selection } \\
\text { method }\end{array}$ & $\begin{array}{c}\text { mutation } \\
\text { probability }\end{array}$ & $\begin{array}{c}\text { selection } \\
\text { probability }\end{array}$ & $\begin{array}{c}\text { evolutionary } \\
\text { generations }\end{array}$ \\
\hline case 1 & binary & 10 -bit & 60 & roulette & 0.1 & 0.6 & 100 \\
case 2 & binary & 10 -bit & 60 & roulette & 0.2 & 0.7 & 300 \\
case 2 & binary & 10 -bit & 60 & roulette & 0.2 & 0.7 & $100 \times 3$ \\
\hline
\end{tabular}

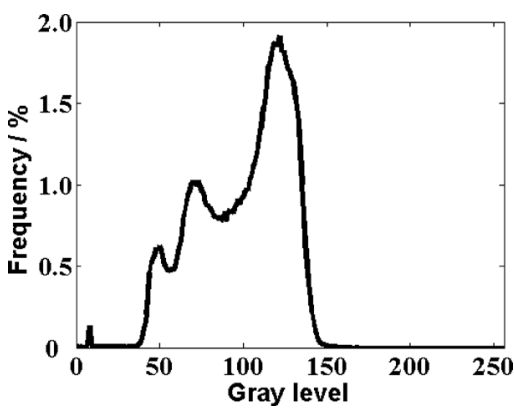

Fig. 5. Gray-level histogram of micrograph.

Table 4. Computational proportions of the mineralogical phases.

\begin{tabular}{ccccccc}
\hline $\begin{array}{c}\text { mineralogical } \\
\text { phases }\end{array}$ & magnetite & hematite & fayalite & $\begin{array}{c}\text { calcium } \\
\text { silicate }\end{array}$ & $\begin{array}{c}\text { calcium } \\
\text { ferrite }\end{array}$ & pore \\
\hline case 1 & 57.36 & 1.62 & 20.84 & 15.33 & 0.85 & 4.37 \\
case 2 & 55.15 & 4.84 & 21.47 & 10.58 & 0.07 & 7.89 \\
case 3 & 54.44 & 3.69 & 23.07 & 9.45 & 0.84 & 8.51 \\
\hline
\end{tabular}

the results are listed separately in Table 4.

\subsection{Case One}

In this case, the number of evolutionary generations was fixed at 100. Figure 6 shows that the difference varies with the evolutionary generation. The final difference between the computational result and the actual gray-level histogram was 0.1884 .

In Fig. 6, the average difference of the population and the minimum difference of the final population are plotted. The average difference decreased as the evolutionary generation increased, especially before the 10th generation. The average difference decreased from 0.68 to about 0.6. However, the rate of decrease became consistently lower as the generation number increased. After the 35 th generation, the minimum difference did not change, meaning that the diversity of the population was minimal after 10 generations. This reduced diversity was due to the low rate of mutations in each generation (only 0.1 ), as was the selection probability (only 0.6 ). In these conditions, $60 \%$ of individuals in the population are selected for the next generation, and $10 \%$ were selected for the mutation. Therefore, most individuals were identical after several generations.

Comparison of the final solution in Table 4 with Fig. 4 indicates that the fraction of pores in the solution was too small, only $4.37 \%$. Figure 4 shows several large areas of pores; therefore, the proportion should have been higher.

\subsection{Case Two}

In this case, some parameters were revised based on the case 1 . The mutation probability was fixed at 0.2 , a little higher than that in case 1, and the selection probability was increased to 0.7 . The evolutionary generations were fixed at

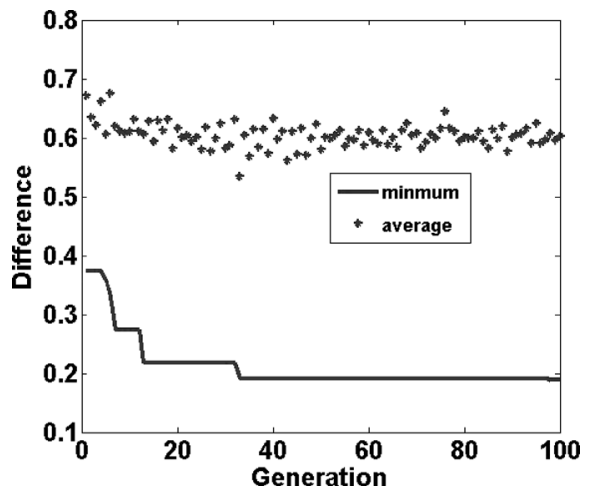

Fig. 6. Computational difference dependent on the generation.

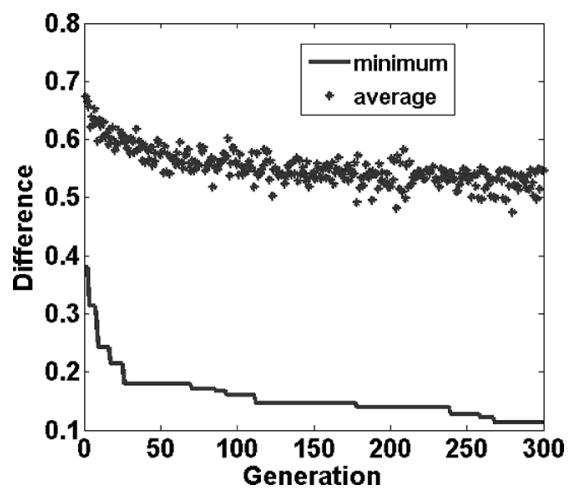

Fig. 7. Computational difference dependent on the generation.

300. Figure 7 shows that the difference varied with the evolutionary generation. The final difference between the computational result and the actual gray-level histogram was 0.1129 .

Figure 7 shows that the average difference among the population decreased more quickly from 0.68 to about 0.5 . The minimum difference among the generation also decreased quickly, from 0.38 to 0.11 . After 100 generations, the minimum difference was less than 0.15 , and the average difference was relatively constant. Due to the higher mutation probability, the minimum difference decreased continuously. A comparison of the results of case two with that of case one shows that the fraction of pores increased from 4.37 to $7.89 \%$, and the fractions of both magnetite and calcium silicate decreased.

\subsection{Case Three}

In this case, an improved genetic algorithm ${ }^{6}$ was adopted. Because the standard genetic algorithm performed well in case two, with the mutation probability fixed at 0.2 and a selection probability fixed at 0.7 were fixed, these two parameters were fixed at the same levels. The generations, however, were divided in this case into three sets of 100 . The solution with minimum difference in the first 100 generations became the beginning point for the next 100 gener- 
ations. The points near this beginning point were calculated. With this method, the population was not trapped in a local minimum. Figure 8 shows that the difference varied with the evolutionary generation. The final difference between the computational result and the actual gray-level

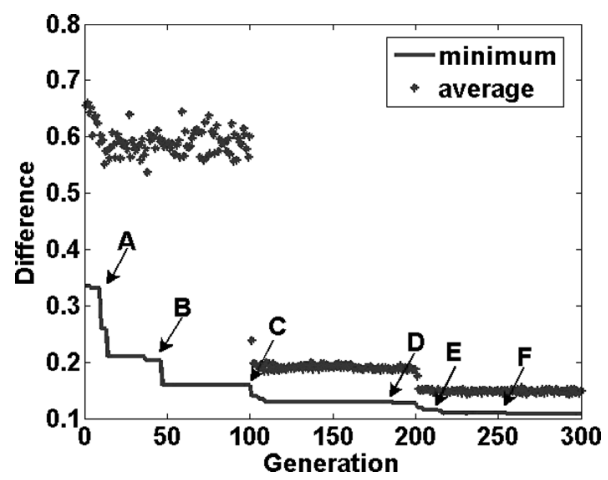

Fig. 8. Computational difference dependent on the generation. histogram was 0.1082 .

As shown in Fig. 8, the average difference, depending on the generation, could be divided into 3 sets. In the first set, the average difference decreased from 0.68 to 0.55 , and the minimum difference decreased from 0.34 to 0.14 . In the second set, the average difference of the population was about 0.18 , and the minimum difference decreased from 0.14 to 0.12 . In the third set, the average difference was about 0.14 , and the minimum difference decreased from 0.12 to 0.10 . The population in the second and the third sets is distributed near the point representing minimum difference in the first step and second step respectively.

Comparison of the results of these three cases demonstrates that the fraction of pores increased as the difference decreased. Similarly, the fraction of magnetite increased as the difference decreased. Some special solutions, marked $\mathrm{A}-\mathrm{H}$ in Fig. 8, are plotted together for comparison, as shown in Fig. 9. This analysis indicates that the fitness of the solution increased as the difference decreased. The peak

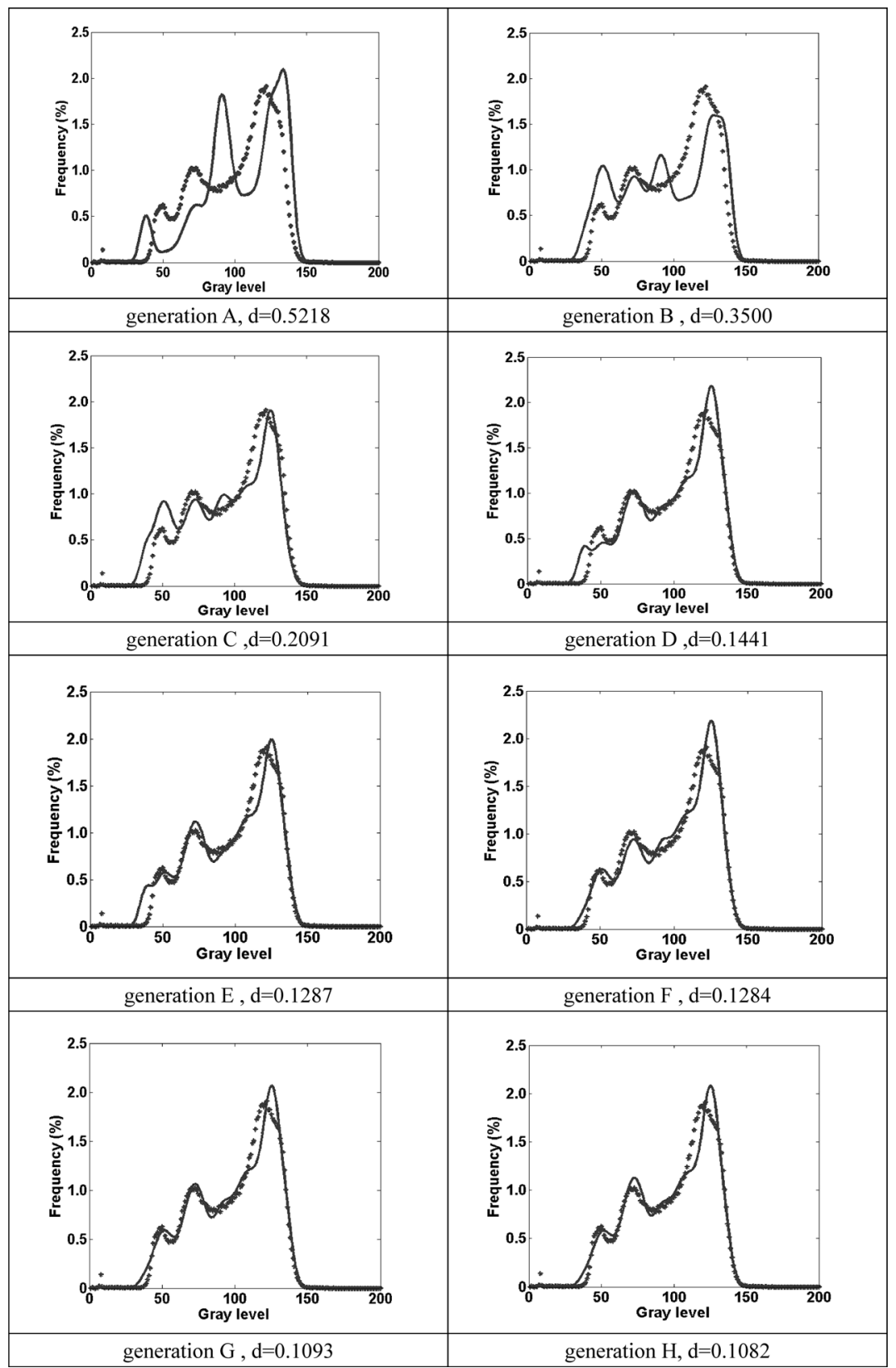

Fig. 9. Fitness dependent on the generation. 
of hematite (gray level 137) in Generation C, for example, was lower than that in Generation F. Therefore, the fraction of hematite in the final solution of case 1 was $1.62 \%$, whereas that of case 2 was $4.84 \%$. Similarly, the peak of hematite in the generation $\mathrm{H}$, was slightly lower than that in generation $\mathrm{F}$; therefore, the fraction of hematite in case 3 was $3.69 \%$, also slightly lower than that in case 2 . The solutions of these cases agree with the fitness.

Case three demonstrates that the main mineralogical phases were magnetite, slag (such as fayalite and calcium silicate), and the pores. Their fractions were $54.44 \%$, $32.52 \%(23.07 \%, 9.45 \%)$, and $8.51 \%$ respectively. Considered in relation to Fig. 4, this method is valid. The total fraction of other phases is about $4.5 \%$, which cannot be determined by the image segment and erosion method.

\section{Conclusions}

These three cases demonstrate that:

(1) The model developed here to quantify the composition of mineralogical phases is valid, and the solution is credible.
(2) The improved genetic algorithm and its related parameters are suitable for calculating the mineralogical phases in iron ore sinter.

(3) This model can be used to calculate the minor mineralogical phases, which cannot be calculated with other methods.

\section{Acknowledgements}

The authors are especially grateful to the Chongqing University Postgraduates' Science and Innovation Fund (200701Y1B0020188).

\section{REFERENCES}

1) D. Jeulin: Proc. of the 42nd Ironmaking Conf., AIME, (1983), 31.

2) D. Jeulin: Ironmaking Steelmaking, 10 (1983), No. 4, 145.

3) T. Shibuya, H. Yanaka and K. Takemoto: Trans. Iron Steel Inst. Jpn., 25 (1985), 257.

4) X. Lv, C. Bai, G. Qiu, M. Hu and S. Zhang: ISIJ Int., 48 (2008), 186.

5) J. H. Holland: SIAM J. Comput., 2 (1973), No. 2, 88.

6) V. Togan and A. T. Daloglu: Comput. \& Struct., 86 (2008), No. $11-12,1204$. 